\title{
Hematopoietic stem cell transplantation for cytidine triphosphate synthase 1 (CTPS1) deficiency
}

DOI:

10.1038/s41409-018-0246-x

\section{Document Version}

Accepted author manuscript

Link to publication record in Manchester Research Explorer

\section{Citation for published version (APA):}

Nademi, Z., Wynn, R., Slatter, M., Hughes, S., Bonney, D., Qasim, W., Latour, S., Trück, J., Patel, S., Abinun, M., Flood, T., Hambleton, S., Cant, A. J., Gennery, A. R., \& Arkwright, P. (2018). Hematopoietic stem cell transplantation for cytidine triphosphate synthase 1 (CTPS1) deficiency. Bone marrow transplantation. https://doi.org/10.1038/s41409-018-0246-x

\section{Published in:}

Bone marrow transplantation

\section{Citing this paper}

Please note that where the full-text provided on Manchester Research Explorer is the Author Accepted Manuscript or Proof version this may differ from the final Published version. If citing, it is advised that you check and use the publisher's definitive version.

\section{General rights}

Copyright and moral rights for the publications made accessible in the Research Explorer are retained by the authors and/or other copyright owners and it is a condition of accessing publications that users recognise and abide by the legal requirements associated with these rights.

\section{Takedown policy}

If you believe that this document breaches copyright please refer to the University of Manchester's Takedown Procedures [http://man.ac.uk/04Y6Bo] or contact uml.scholarlycommunications@manchester.ac.uk providing relevant details, so we can investigate your claim.

\section{OPEN ACCESS}




\section{Hematopoietic stem cell transplantation for cytidine triphosphate synthase 1 (CTPS1) deficiency}

2 Zohreh Nademi, MD, PhD ${ }^{1,2}$, Robert F. Wynn, MD ${ }^{3}$, Mary Slatter, MD ${ }^{1,2}$, Stephen M. Hughes, PhD ${ }^{3}$, Denise Bonney, MD, PhD 3 ,

3 Waseem Qasim, MD ${ }^{4}$, Sylvain Latour, $\mathrm{PhD}^{5}$, Johannes Trück, MD, DPhil ${ }^{6}$, Smita Patel, PhD ${ }^{7}$, Mario Abinun, MD ${ }^{1,2}$, Terry Flood,

$4 \quad \mathrm{MD}^{1}$, Sophie Hambleton, DPhil ${ }^{1,2}$, Andrew J. Cant, MD ${ }^{1,2}$, Andrew R. Gennery, MD ${ }^{1,2}$, Peter D. Arkwright, MD, PhD

$5 \quad{ }^{1}$ Great North Children's Hospital, Newcastle upon Tyne Hospitals NHS Foundation Trust, UK

$6 \quad{ }^{2}$ Primary Immunodeficiency Group, Institute of Cellular Medicine, Newcastle upon Tyne University, UK

7 3 University of Manchester, Royal Manchester Children's Hospital, Manchester, UK

$8{ }^{4}$ Department of Pediatric Immunology, Great Ormond Street Hospital for Children NHS Foundation Trust, London, UK

$9 \quad{ }^{5}$ Laboratory of Lymphocyte Activation and susceptibility to EBV infection, Inserm UMR 1163, Hôspital Necker-Enfants, Paris,

10 France

$11{ }^{6}$ Division of Immunology and the Children's Research Center, University Children's Hospital, University of Zurich, Zurich,

12 Switzerland

$13{ }^{7}$ Oxford NIHR Biomedical Research Centre, Oxford, UK 


\section{Capsule Summary}

19 CTPS1 deficiency causes early onset herpes virus infections particularly with VZV and EBV and there is a high risk of developing

20 lymphoma. We report eleven patients treated by hematopoietic stem cell transplantation, eight of whom survived with resolution of 21 symptoms.

22

23 Key word: CTPS1, primary immune deficiency, hematopoietic stem cell transplantation, EBV, lymphoma

Word Count: 944 


\section{To the Editor,}

27 Cytidine triphosphate (CTP) synthase 1 is responsible for the catalytic conversion of uridine triphosphate (UTP) to CTP in 28 lymphocytes. ${ }^{1}$ This reaction is important for the biosynthesis of nucleic acids, playing a key role in lymphocyte function and turnover $^{1-2}$. Lack of this enzyme due to mutation in CTPS gene is associated with impaired capacity of activated T and Blymphocytes to proliferate in response to antigen-induced activation. Patients with this mutation all have ancestors from the North West of England ${ }^{1}$ and present in the first decade of life with severe acute and chronic herpes virus infections and recurrent infections with encapsulated bacteria. There is a high risk of EBV-driven lymphoma. ${ }^{1}$ This is a potentially life-threatening primary immune deficiency (PID) and long-term survival has not been reported.

We report on 11 patients with CTPS1 mutations who underwent hematopoietic stem cell transplant (HSCT) at three UK centres (London, Manchester and Newcastle). The molecular diagnosis was made pre-HSCT in six patients and retrospectively in five patients who had undergone HSCT for previously undiagnosed life-threatening PID. All patients had the same homozygous NM_001905.3: c.1692-1G>C mutation (rs145092287) mutation and all the parents were heterozygous carriers. This mutation is known to lead to an abnormal transcript lacking exon 18 and complete lack of protein expression. ${ }^{1}$ Six patients had an affected sibling. Patient 1 had 2 affected siblings who died of infection and hemophagocytic lymphohistiocytosis (HLH) in early childhood and patient 2 also had a sibling who died of overwhelming VZV and EBV infections before diagnosis. Pre-transplant features are summarized in Table 1. Systemic viral infections were seen in all patients, with acute and chronic EBV infections in nine patients, 
42 four suffering from EBV-driven lymphoproliferative disease (LPD) (two CNS and one lung) and one with EBV-driven HLH. Four

43 patients had severe chickenpox. Five patients presented with chronic diarrhea.

Four patients with EBV-driven LPD received rituximab and three EBV-specific cytotoxic T-lymphocytes (CTLs) (two pretransplant and one post-transplant). The age of the patients at transplant ranged from 15 months to 17 years. The source of stem cells was peripheral blood (PB) in seven patients, bone marrow (BM) in three and cord blood (CB) in one. Eight received matched

$47(10 / 10)$ donor stem cells, two had 1/10 mismatches and one received a haploidentical 6/10 transplant from his father as there were 48 no suitable matched donor (Table 2). All but one received conditioning with fludarabine in combination with treosulfan ( $\mathrm{n}=3$ ),

49 melphalan $(n=4)$, treosulfan and thiotepa $(n=1 \text {, the non-reduced intensity conditioning (RIC) protocol) or low dose busulfan ( } n=2)^{3}$,

50 one received treosulfan and cyclophosphamide. All but one patient received serotherapy with alemtuzumab ( $n=8)$ or anti $T$ cell

51 globulin $(A T G)(n=2)$. CD34 ${ }^{+}$cell dose ranged from $0.4-16.1 \times 10^{6} / \mathrm{kg}$. Neutrophil engraftment $\left(\geq 0.5 \times 10^{9} / \mathrm{L}\right)$ occurred between day

52 11- 23 post-transplant. Ten had $100 \%$ CD3 donor engraftment and one had $76 \%$ in last follow up. immunosuppression and six are off immunoglobulin replacement therapy. Patient 4 died 76 days post-HSCT following EBV CNS reactivation leading to obstructive hydrocephalus. She was treated with external ventricular drainage and three doses of $2 \times 10^{6}$ EBV-CTLs but died of multi-organ failure ${ }^{4}$. Patient 7 developed intractable gut inflammation due to a combination of adenovirus and CMV colitis and chronic graft versus host disease (cGvHD) and died at day +290 despite treatment with ganciclovir and 
cidofovir for the viral infections and corticosteroids, ciclosporin, tacrolimus and infliximab as anti-inflammatory drugs. Patient 8 developed mild acute skin GvHD post-transplant, but later suffered from intractable chronic skin GvHD (but no gut or liver disease) unresponsive to corticosteroids and extracorporeal photophoresis. Twelve months post-transplantation he developed a tremor and altered mental state progressing to coma and died 15 months post-transplant. Brain biopsy and magnetic resonance (MR) scan performed prior to death were indicative of progressive multifocal leukoencephalopathy (PML), although no JC, BK or astrovirus were isolated from cerebrospinal fluid (CSF) or brain tissue.

CTPS1 deficiency is a serious life-limiting immunodeficiency with a high risk of death from VZV and EBV in the first decade of life. Seven (41\%) of 17 patients worldwide with CTPS1 deficiency have died, three post and four prior to HSCT (one from fulminant VZV and the other three from fulminant EBV LPD). These deaths were prior to clinical availability of rituximab. However, mild clinical phenotype has previously been described ${ }^{5}$. HSCT can be curative but is not without potential risks and complications. Kucuk et al also reported two siblings with this mutation who had successful transplantation ${ }^{6}$. The current report is the largest series of children with CTPS1 deficiency who have undergone HSCT. The overall survival post-HSCT in this series was $72 \%$ which

70 is slightly lower than seen after HSCT for other combined PIDs ${ }^{7}$. Pre-transplantation, extra-nodal EBV-driven LPD of the brain and lung as well as fulminant VZV pneumonitis requiring ventilation in intensive care are major risks. The post-transplantation course can be challenging to even the specialist centres because of re-activation of EBV and other herpes viruses, particularly in the brain

73 and gastrointestinal tract, but also because of the risk of unusually severe skin and gut cGvHD, the latter which may be partly 
fuelled by ongoing underlying low grade chronic viral infection. Both patients that developed fatal GvHD had transplants from $9 / 10$

75 matched unrelated donors. Viral reactivation can be observed prior to donor T-lymphocyte engraftment post-transplant and perhaps

76 virus-specific CTLs may be helpful in some of these patients. There is no doubt that HSCT can be curative in this condition and we

77 recommend early transplantation before the onset of end-organ damage and for patients without fully matching family or unrelated

78 donors, the consideration of ex-vivo T-cell depletion strategies.

79 The authors confirm there is no conflict of interest.

80 Zohreh Nademi ${ }^{1,2}$

81 Robert F. Wynn ${ }^{3}$

82 Mary Slatter ${ }^{1,2}$

83 Stephen M. Hughes ${ }^{3}$

84 Denise Bonney ${ }^{3}$

85 Waseem Qasim ${ }^{4}$

86 Sylvain Latour ${ }^{5}$ 
Mario Abinun 1,2

Terry Flood ${ }^{1}$

91 Sophie Hambleton ${ }^{1,2}$

93 Andrew R. Gennery ${ }^{1,2}$

94 Peter D. Arkwright ${ }^{3}$

$95{ }^{1}$ Great North Children's Hospital, Newcastle upon Tyne Hospitals NHS Foundation Trust, UK

96 2Primary Immunodeficiency Group, Institute of Cellular Medicine, Newcastle upon Tyne University, UK

97 3University of Manchester, Royal Manchester Children's Hospital, Manchester, UK ${ }^{4}$ Department of Pediatric Immunology, Great Ormond Street Hospital for Children NHS Foundation Trust, London, UK 
${ }^{7}$ Department of Immunology, John Radcliffe Hospital, Oxford, UK

\section{References}

1. Martin E, Palmic N, Sanquer S, Lenoir C, Hauck F, Mongellaz C et al. CTP synthase 1 deficiency in humans reveals its central role in lymphocyte proliferation. Nature 2014; 510:288-292.

2. Wynn RF, Arkwright PD, Haque T, Gharib M, Wilkie G, Morton-Jones M et al. Treatment of Epstein-Barr-virus-associated

$$
\text { 6:344-46. }
$$

3. Güngör T, Teira P, Slatter M, Stussi G, Stepensky P, Moshous D et al. Reduced-intensity conditioning and HLA-matched haemopoietic stem-cell transplantation in patients with chronic granulomatous disease: a prospective multicentre study. Lancet 2014; 383:436-48.

4. Naik S, Nicholas S, Martinez C, Leen A, Hanley P, Gottschalk S et al. Adoptive Immunotherapy for Primary Immunodeficiency Disorders with Virus-Specific Cytotoxic T-lymphocytes. J Allergy Clin Immunol 2016; 137: 1498-1505. 
5. Trück J, Kelly DF, Taylor JM, Kienzler A, Lester T, Seller A et al. Variable phenotype and discrete alterations of immune phenotypes in CTP synthase 1 deficiency: Report of 2 siblings. J Allergy Clin Immunol, 2016; Volume 138, Issue 6, Pages 1722-1725.

6. Kucuk ZY, Zhang K, Filipovich L, Blessing J. CTP Synthase 1 Deficiency in Successfully Transplanted Siblings with Combined Immune Deficiency and Chronic Active EBV Infection. J Clin Immunol. 2016 Nov; 36(8):750-753.

7. Dvorak CC, Cowan MJ. Hematopoietic stem cell transplantation for primary immunodeficiency disease. Bone Marrow Transplant. 2008 Jan; 41(2):119-26. 


\section{Table 1 Patient characteristics}

\begin{tabular}{|c|c|c|c|c|c|c|c|c|c|c|c|c|c|}
\hline \multirow[t]{2}{*}{ Patient } & \multirow[t]{2}{*}{ Sex } & \multicolumn{6}{|c|}{ Clinical history } & \multirow[b]{2}{*}{$\underline{\text { IPI }}$} & \multirow[b]{2}{*}{ Iymphoma } & \multirow{2}{*}{$\begin{array}{l}\text { Family } \\
\text { History }\end{array}$} & \multirow{2}{*}{$\begin{array}{l}\text { lymphocytes } \\
\text { subset (x10^9/L) }\end{array}$} & \multirow{2}{*}{$\begin{array}{l}\text { Immunoglobulin } \\
\text { Normal range }(\mathrm{g} / \mathrm{L}) \\
\operatorname{lgG}(3.7-15.8) \\
\lg \mathrm{A}(0.3-1.3) \\
\lg \mathrm{M}(0.5-2.2)\end{array}$} & \multirow{2}{*}{$\begin{array}{l}\text { Age at } \\
\text { presentation }\end{array}$} \\
\hline & & SPI & EBV-LPD & $\underline{\mathrm{S} \text { VZV }}$ & Chronic Diarrhoea & $\underline{\mathrm{HLH}}$ & Viral viremia & & & & & & \\
\hline Pt1 & $M$ & $\bar{Y}$ & $\mathrm{~N}$ & $\bar{N}$ & $\mathrm{Y}(\mathrm{HHV} 6)$ & $\bar{N}$ & $\mathrm{~N}$ & $\mathrm{~N}$ & $\mathrm{~N}$ & $\mathrm{Y}$ & $\begin{array}{l}\text { CD19 0.3, CD3 2.2, } \\
\text { CD4 0.9, CD8 1.2, } \\
\text { NK } 0.49\end{array}$ & $\begin{array}{l}\lg G 19.1, \lg A 0.5 \\
\lg M 0.84\end{array}$ & 4 months \\
\hline Pt2 & $\mathrm{F}$ & $\mathrm{N}$ & $\mathrm{N}$ & Y & $\mathrm{N}$ & $\mathrm{N}$ & $\mathrm{Y}(\mathrm{EBV})$ & $\mathrm{N}$ & $\mathrm{N}$ & $\mathrm{Y}$ & $\begin{array}{l}\text { CD19 1.1, CD3 1.9, } \\
\text { CD4 1.4, CD8 0.37, } \\
\text { NK } 0.21\end{array}$ & $\begin{array}{l}\lg G 5.5, \lg A 2.5 \\
\lg M 0.5\end{array}$ & 17 months \\
\hline Pt3 & M & $\begin{array}{l}Y \\
(A D V)\end{array}$ & Y & $\mathrm{N}$ & Y (Norovirus) & $\mathrm{N}$ & Y (EBV) & $\mathrm{N}$ & $\mathrm{Y}(\mathrm{CNS})$ & $\mathrm{N}$ & $\begin{array}{l}{ }^{\wedge} \mathrm{CD} 190, \mathrm{CD} 30.47 \\
\mathrm{CD} 40.43, \mathrm{CD} 8 \\
0.02, \mathrm{NK} 0.03\end{array}$ & $\begin{array}{l}\lg G 4.8, \lg A 4.4 \\
\lg M 0.8\end{array}$ & 5 months \\
\hline Pt4 & $\mathrm{F}$ & $\mathrm{N}$ & Y & Y & $\mathrm{N}$ & Y & $\mathrm{N}$ & $\mathrm{N}$ & $\mathrm{N}$ & $\mathrm{N}$ & $\begin{array}{l}{ }^{\wedge} \mathrm{CD} 190.01, \mathrm{CD} 3 \\
0.52, \mathrm{CD} 40.25 \\
\text { CD8 0.25, NK } 0.03\end{array}$ & $\begin{array}{l}\lg G 7, \lg A 0.46 \\
\lg M 0.39\end{array}$ & 6 years \\
\hline${ }^{*} \mathrm{Pt5}$ & $\mathrm{F}$ & $\begin{array}{l}Y \\
\text { (Hinf) }\end{array}$ & $\begin{array}{l}Y \\
\text { (CNS) }\end{array}$ & Y & $\mathrm{N}$ & $\mathrm{N}$ & $\mathrm{N}$ & $\mathrm{N}$ & $\mathrm{N}$ & $\mathrm{Y}$ & $\begin{array}{l}{ }^{\wedge} \mathrm{CD} 19 \quad 0.13, \mathrm{CD} 3 \\
0.46, \mathrm{CD} 40.28 \\
\mathrm{CD} 80.18, \mathrm{NK} 0.02\end{array}$ & $\begin{array}{l}\lg G 10.9, \lg A \\
2.01, \lg M 0.59\end{array}$ & 1 month \\
\hline${ }^{*}$ Pt6 & M & $\begin{array}{l}Y \\
\text { (Hinf) }\end{array}$ & $\mathrm{N}$ & $\mathrm{N}$ & $\mathrm{N}$ & $N$ & $\mathrm{Y}(\mathrm{EBV})$ & $\mathrm{N}$ & $\mathrm{N}$ & $\mathrm{Y}$ & $\begin{array}{l}\text { CD19 0.17, CD3 } \\
0.99, \text { CD4 0.57, } \\
\text { CD8 0.48, NK } 0.12\end{array}$ & $\begin{array}{l}\lg G 6.41, \lg A \\
0.42, \lg M 0.47\end{array}$ & 3 years \\
\hline${ }^{\star \star} \mathrm{Pt} 7$ & $\mathrm{~F}$ & Y & $\mathrm{N}$ & $\mathrm{N}$ & Y & $\mathrm{N}$ & Y (EBV) & $\mathrm{N}$ & $\mathrm{N}$ & Y & CD19 1.01, CD3 & $\lg G$ 24.7, $\lg A$ 0.6, & 7 months \\
\hline
\end{tabular}




\begin{tabular}{|c|c|c|c|c|c|c|c|c|c|c|c|c|c|}
\hline & & (EBV) & & & & & & & & & 4.39, CD4 1.41, & $\lg M 0.84$ & \\
\hline **Pt8 & M & $\begin{array}{l}Y \\
\text { (EBV) }\end{array}$ & $\mathrm{N}$ & $\mathrm{N}$ & $\mathrm{Y}$ & $\mathrm{N}$ & $\mathrm{Y}(\mathrm{EBV})$ & $\mathrm{N}$ & $\mathrm{N}$ & $\mathrm{Y}$ & $\begin{array}{l}\text { CD19 0.65, CD3 } \\
1.68, \text { CD4 0.83, } \\
\text { CD8 0.78, NK } 0.05\end{array}$ & $\begin{array}{l}\lg G 12.4, \lg A \\
0.66, \lg M 0.12\end{array}$ & 14 months \\
\hline Pt9 & M & Y & $\mathrm{N}$ & $\mathrm{N}$ & $Y$ & $\mathrm{~N}$ & $\mathrm{Y}(\mathrm{EBV})$ & $Y$ & $\mathrm{~N}$ & $\mathrm{~N}$ & $\begin{array}{l}\text { CD19 0.25, CD3 } \\
1.8, \text { CD4 1.1, CD8 } \\
0.62, \text { NK } 0.15\end{array}$ & $\begin{array}{l}\lg G 5.34, \lg A \\
1.01, \lg M 0.62\end{array}$ & 2 months \\
\hline Pt10 & $\mathrm{F}$ & $\mathrm{Y}$ & $\mathrm{N}$ & $\mathrm{Y}$ & $\mathrm{N}$ & $\mathrm{N}$ & $\mathrm{N}$ & $\mathrm{N}$ & $\mathrm{N}$ & $\mathrm{N}$ & $\begin{array}{l}\text { CD19 0.4, CD3 3.7, } \\
\text { CD4 1.3, CD8 2.3, } \\
\text { NK } 0.09\end{array}$ & $\begin{array}{l}\lg G 16.70, \lg A \\
0.45, \lg M 0.24\end{array}$ & 7 years \\
\hline Pt11 & M & Y & $\begin{array}{l}\text { Y } \\
\text { (LUNG) }\end{array}$ & $\mathrm{Y}$ & $\mathrm{N}$ & $\mathrm{N}$ & $\mathrm{N}$ & $\mathrm{N}$ & $\mathrm{N}$ & $\mathrm{N}$ & $\begin{array}{l}\text { CD19 0.3, CD3 2.8, } \\
\text { CD4 1.7, CD8 0.9, } \\
\text { NK } 0.2\end{array}$ & $\begin{array}{l}\lg G 16.5, \lg A \\
1.45, \lg M 1.10\end{array}$ & 2 years \\
\hline
\end{tabular}

Pt: Patient; M: Male; F: Female; LPD: Lymphoproliferative disease; EBV: Epstein Bar virus; VZV: varicella zoster virus; ADV:

Adenovirus; HHV6: Human Herpes Virus 6; Hinf: Haemophilus influenzae; SPI: sinopulmonary infection; S VZV: Severe VZV infection i.e. pneumonitis; IPI: Invasive Pneumococcal Infection; HLH: Hemophagocytic Lymphohistiocytosis; Y: Yes; N: No.

*siblings from Family 1

** siblings from family 2

${ }^{\wedge}$ patients who had abnormal lymphocyte subsets 
Table 2: Details of hematopoietic stem cell transplantation

\begin{tabular}{|c|c|c|c|c|c|c|c|c|c|c|c|c|}
\hline Patient & $\begin{array}{l}\text { Age@ } \\
\text { HSCT } \\
\text { (Year) }\end{array}$ & Donor & Source & Conditioning & $\begin{array}{l}\text { GvHD } \\
\text { Prophylaxis }\end{array}$ & $\begin{array}{l}\text { Neutrophil/CD3 } \\
\text { engraftment }^{\star \star \star}\end{array}$ & $\begin{array}{l}\text { Viral } \\
\text { reactivation } \\
\text { Timing }\end{array}$ & $\begin{array}{l}\text { Chimerism \% } \\
\text { donor }\end{array}$ & $\begin{array}{l}\text { GvHD } \\
\text { Site/grade }\end{array}$ & Off IVIG & $\begin{array}{l}\text { Outcome } \\
\text { Time of F/U }\end{array}$ & Reason for death \\
\hline Pt 1 & 17 & $\begin{array}{l}\text { MUD } \\
10 / 10\end{array}$ & PBSC & $\begin{array}{l}\text { Mel 140/Flu } \\
\text { 150/Alem } 1\end{array}$ & CSA/MMF & $D+13 /+45$ & $\begin{array}{l}\mathrm{CMV} / \mathrm{HHV} 6 \\
\mathrm{D}+37 /+34\end{array}$ & $\begin{array}{l}\text { CD15 93\% } \\
\text { CD19 97\% } \\
\text { CD3 100\% }\end{array}$ & Skin, GI & $\bar{Y}$ & $\begin{array}{l}A \& W \\
12 y\end{array}$ & \\
\hline Pt 2 & 8 & $\begin{array}{l}\text { MUD } \\
10 / 10\end{array}$ & BM & $\begin{array}{l}\text { Treo 42/Cyclo } \\
\text { 200/Alem } 1\end{array}$ & CSA/MMF & $D+14 /+60$ & $\begin{array}{l}\text { ADV } \\
\text { D0 }\end{array}$ & $\begin{array}{l}\text { CD15 25\% } \\
\text { CD19 48\% } \\
\text { CD3 76\% }\end{array}$ & Skin GII & $\mathrm{Y}$ & $\begin{array}{l}A \& W \\
8 y\end{array}$ & \\
\hline Pt 3 & 1 & $\begin{array}{l}\text { MUD } \\
10 / 10\end{array}$ & CB & $\begin{array}{l}\text { Treo 42/Flu } \\
150\end{array}$ & CSA/MMF & $D+23 /+17$ & $\begin{array}{l}\text { EBV/ADV } \\
\text { D0 }\end{array}$ & WB $100 \%$ & $\begin{array}{l}\text { Skin GIII } \\
\text { Gut GI }\end{array}$ & Y & $\begin{array}{l}\text { hemiplegia, } \\
\text { developmental } \\
\text { delay, } 4 y\end{array}$ & \\
\hline Pt 4 & 8 & $\begin{array}{l}\text { MUD } \\
10 / 10\end{array}$ & PBSC & $\begin{array}{l}\text { Treo 42/Flu } \\
\text { 150/Alem } 1\end{array}$ & CSA/MMF & $D+14 /+46$ & $\begin{array}{l}\text { EBV } \\
\text { D+39 }\end{array}$ & WB $100 \%$ & Skin Gll & $\mathrm{Y}$ & died & $\begin{array}{l}\text { EBV } \\
\text { encephalitis, } \\
\text { D+76 }\end{array}$ \\
\hline${ }^{*}$ Pt 5 & 9 & $\begin{array}{l}\text { MUD } \\
10 / 10\end{array}$ & PBSC & $\begin{array}{l}\text { Mel 140/Flu } \\
\text { 150/Alem } 1\end{array}$ & CSA & $D+13 /+180$ & $\begin{array}{l}\text { EBV } \\
\text { D+87 }\end{array}$ & WB $100 \%$ & No & Y & $\begin{array}{l}\text { A\&W } \\
22 y\end{array}$ & \\
\hline${ }^{*}$ Pt 6 & 5 & $\begin{array}{l}\text { MUD } \\
10 / 10\end{array}$ & PBSC & $\begin{array}{l}\text { Treo 42/Flu } \\
\text { 150/Alem } 1\end{array}$ & CSA/MMF & $D+11 /+59$ & $\mathrm{~N} / \mathrm{A}$ & WB $100 \%$ & No & $\mathrm{Y}$ & $\begin{array}{l}A \& W \\
17 y\end{array}$ & \\
\hline${ }^{\star \star P t} 7$ & 8 & $\begin{array}{l}\text { MUD } \\
9 / 10\end{array}$ & PSBC & $\begin{array}{l}\text { Mel 140/Flu } \\
\text { 150/Alem } 1\end{array}$ & CSA/MMF & $\mathrm{D}+11 / \mathrm{NA}^{\wedge}$ & $\begin{array}{l}\text { CMV/ADV } \\
D+31 /+71\end{array}$ & WB $100 \%$ & Gut GIII & $\mathrm{N} / \mathrm{A}$ & died & $\begin{array}{l}\text { chronic, } \\
\text { intractable CMV } \\
\text { \& adenovirus } \\
\text { enteritis with } \\
\text { secondary gut } \\
\text { cGvHD, D+290 }\end{array}$ \\
\hline${ }^{\star \star}$ Pt 8 & 12 & $\begin{array}{l}\text { MUD } \\
9 / 10 \mathrm{DQ}\end{array}$ & BM & $\begin{array}{l}\text { Bu } 9.5 \text { (target } \\
\text { AUC 60, RIC)/ } \\
\text { Flu 180/Alem }\end{array}$ & CSA/MMF & $D+15 /+88$ & $\begin{array}{l}\text { EBV } \\
D+248^{\wedge \wedge}\end{array}$ & WB $100 \%$ & Skin GIII & $\mathrm{Y}$ & died & $\begin{array}{l}\text { intractable skin } \\
\text { cGvHD, } \\
\text { CNS PML, }\end{array}$ \\
\hline
\end{tabular}




\begin{tabular}{|c|c|c|c|c|c|c|c|c|c|c|c|}
\hline Pt 9 & 5 & $\begin{array}{l}\text { MUD } \\
10 / 10\end{array}$ & PBSC & $\begin{array}{l}\text { Mel 140/Flu } \\
\text { 150/Alem } 1\end{array}$ & CSA/MMF & $D+17 /+9 m$ & $\begin{array}{l}\text { BK virus } \\
D+7\end{array}$ & $\begin{array}{l}\text { CD15 85\% } \\
\text { CD3 100\% }\end{array}$ & Skin GII & Y & $\begin{array}{l}\text { A\&W } \\
1 y\end{array}$ \\
\hline Pt 10 & 13 & $\begin{array}{l}\text { MSD } \\
10 / 10\end{array}$ & BM & $\begin{array}{l}\text { Bu (target to } \\
\text { AUC 60, RIC)/ } \\
\text { Flu 180/ATG } \\
40\end{array}$ & & $D+40 /+102$ & $\mathrm{~N} / \mathrm{A}$ & WB 100\% & No & No & $\begin{array}{l}\text { A\&W } \\
9 m\end{array}$ \\
\hline Pt 11 & 3 & $\begin{array}{l}\text { Haplo } \\
\text { TCR } \\
\text { a/b } \\
\text { depleted }\end{array}$ & PBSC & $\begin{array}{l}\text { Treo 42/TT } \\
\text { 10/Flu 160/ } \\
\text { ATG15 }\end{array}$ & CSA/MMF & $\mathrm{D}+35 /+34^{\wedge \wedge}$ & $\begin{array}{l}\text { EBV } \\
D+68^{\wedge \wedge}\end{array}$ & WB $100 \%$ & No & No & $\begin{array}{l}A \& W \\
8 m\end{array}$ \\
\hline
\end{tabular}

Pt: Patient; MUD: Matched unrelated donor; MSD: Matched sibling donor; PBSC: Peripheral blood stem cells; BM: Bone marrow; CB: Cord blood; F/U: Follow up; Neut: Neutrophil; GvHD: Graft versus Host Disease; IVIG: Immunoglobulin; LPD: Lymphoproliferative Disease; PML: Progressive Multifocal Leucoencephalopathy; A/W: Alive and Well; D: Day; N/A: Not Applicable; WB: Whole blood; Y: Yes; y: year, m: month; Treo: treosulfan, dose g/m2; TT: thiotepa, dose mg/kg; Bu: busulphan; Mel: melphalan, dose mg/m2; Flu: fludarabine, dose mg/m2: Cyclo: cyclophosphamide, dose mg/kg; Alem: alemtuzumab, dose mg/kg; ATG: anti-thymocyte globulin, dose mg/kg; EBV: Epstein Bar virus; ADV: Adenovirus; HHV6: Human Herpes Virus 6; CMV: cytomegalovirus.

*siblings from Family 1

${ }^{* *}$ siblings from family 2

${ }^{* * *} \mathrm{CD} 3$ engraftment: $\mathrm{CD} 3 \geq 0.3 \times 10^{9} / \mathrm{L}$

$\wedge$ The patient always had $\mathrm{CD} 3>0.3 \times 10^{9} / \mathrm{L}$ due to receiving EBV CTLs

$\wedge \wedge$ The patient was on immunosuppressive treatment 